Proc. Estonian Acad. Sci. Eng., 2001, 7, 4, 289-308

\title{
MODELLING NON-LINEAR SYSTEMS BY EXTENDED FOURIER SERIES
}

\author{
Vello KUKK \\ Department of Automatics, Tallinn Technical University, Ehitajate tee 5, 19086 Tallinn, Estonia; \\ v.kukk@ieee.org \\ Received 2 May 2001, in revised form 20 September 2001

\begin{abstract}
The paper deals with modelling of non-linear systems consisting of highly oscillating subsystems. Signals are represented as Fourier series extended by half-frequency components that make it possible to model the transients. Macromodels for non-linear transformations are based on harmonic linearization, implemented by the Chebyshev expansion. This enables independent description of the non-linear transformation and calculation of the Chebyshev transformation of waveforms that appear during the solution. Integration method based on this approach has excellent
\end{abstract} \\ stability properties and its accuracy can be checked by using different number of harmonics.
}

Key words: modelling, non-linear systems, oscillators, Fourier series, Chebyshev transformation.

\section{INTRODUCTION}

We shall consider systems that exhibit oscillation-like behaviour, mainly conventional oscillators, but other types of narrow band systems are also kept in mind. The main problems that arise when designing or analysing such systems are the following:

1) oscillations with slowly changing parameters need a huge number of time steps to be modelled; it may slow down the modelling process when conventional simulators are used;

2) very high accuracy may be needed; nowadays, quality of oscillators is one of the most important parameters determining the quality of communication systems;

3) conventional oscillators are simple circuits consisting of few components, but modern oscillators have become complicated systems using controllers to satisfy high demands for accuracy both in time and temperature variation; 
4) it has been realized that even classical oscillators may exhibit chaos $\left[{ }^{1}\right]$; chaotic behaviour should be detectable, chaotic oscillations (and, for example, synchronization) need fast modelling.

Numerical methods for solving non-linear systems of differential equations, used in common circuit simulators, are based on integration of differential equations by means of implicit methods that reduce the equations to non-linear algebraic equations. This procedure is repeated for every time-step recursively, changing the time-step and order of the method to achieve maximum gain in time. For oscillating processes, the trapezoidal formula is usually recommended. However, as integration steps are limited both by stability conditions and accuracy control, maximum step must be small compared with the total integration time. For example, using the trapezoidal formula, accuracy of the period equal to $1 \mathrm{ppm}$ requires about 1500 steps per period $\left[{ }^{2}\right]$. Transients appearing during the start-up may last thousands of periods with enormous waste of simulation time. Irscheid has modelled a simple oscillator to obtain steady state using SPICE; it required 36 hours of computer time $\left[^{3}\right]$. At the same time, in most of the neighbouring periods the forms of the signal were rather similar.

Interest in periodically changing circuits has grown significantly last years because of the wide usage of switching circuits like SC-filters, power converters, etc. Those circuits can be modelled as discrete time circuits with very low accuracy, or using complete models that need enormous simulation time. The envelope following method has been reported to be able to solve problems that would need weeks of modelling time using the conventional SPICE simulator $\left[{ }^{4}\right]$.

All special methods for solving such problems are based on the idea of using frequency domain representation for signals and working with signal parameters (amplitude, phase, frequency, etc.) rather than with instant values in the time domain. The most common method is harmonic linearization which assumes that input signal of a non-linear element is sine wave and output signal consists of higher harmonics, most of which are filtered out in linear dynamical part of the system. In the simplest case, only first harmonic is used and many practical problems (determination of limit cycles, their parameters, etc.) have been solved using this method. It has been shown that simple equivalent circuits can be built to make common simulators usable $\left[^{2,5-7}\right]$, but problems may arise because of non-uniqueness of signal expressions. For more complicated systems, various generalizations have been proposed, which use more harmonics, combine time and frequency domain, allow multi-tone excitations, consider transients, etc. [ $\left.{ }^{8,9}\right]$. Anyway, that means simultaneous solution of huge systems of non-linear equations because every variable is represented by a number of parameters.

A new method, based on frequency domain representation of the signal, but with a time domain integration, has been proposed and several aspects of the method have been discussed in $\left.{ }^{10-13}\right]$. In this paper, we shall study in detail the properties of this method referred to in the following as the EFS (Extended Fourier Series) method. 


\section{SIMPLE SIGNAL MODEL}

Assume that signals are represented as

$$
v(t)=A(t) \cos \varphi(t),
$$

where amplitude $A(t)$ and frequency $\varphi(t)=\omega(t)$ are slowly changing functions. The derivative of (1) is

$$
v^{\prime}(t)=A^{\prime}(t) \cos \varphi(t)-A(t) \varphi^{\prime}(t) \sin \varphi(t),
$$

which is assumed to have also slowly changing amplitudes and phases. It follows that we can use a large time-step and have fast modelling. Even if non-linear elements generate higher harmonics, they will also have slow amplitudes and phases and we can proceed with more variables but fast solution.

In the frequency domain, "slow" means that the spectrum of signals is concentrated into narrow bands around fundamental and higher harmonics. Both linear and non-linear transformations preserve this structure of signals.

Although this approach has been widely used, it has an obvious drawback: representation (1) is unique only if narrow-band condition is satisfied. However, it is difficult to satisfy this condition in transients. Bandwidth can be controlled only in rather specific cases. A typical effect that appears can be described as frequency doubling, when $A(t)$ locks not to the low frequency oscillation, but to the doubled frequency:

$$
v(t)=\operatorname{Re}\left[e^{j \Omega t} e^{j \omega t}\right]=\operatorname{Re}\left[e^{j(2 \omega+\Omega) t} e^{-j \omega t}\right] .
$$

Such effects were observed in real simulations and they slowed simulation completely down because of the smaller time-step than needed for the fundamental frequency $\left[{ }^{5}\right]$.

Experience with using harmonic linearization based on fundamental frequency has shown that remarkable acceleration of simulation can be obtained. However, the danger of frequency doubling exists and transients are not modelled correctly in case of fast changes (switching) of the parameters. The last case is important in practice because of the oscillator control that usually switches capacitors to correct frequency.

This experience raised the question of modification of the representation (1) to obtain a unique expression for signals that makes it possible to take into account higher harmonics, but at the same time accelerates simulation. That means keeping attention on the oscillating processes, but also providing an acceptable modelling of transients. 


\section{GENERALIZED PHASE}

In $\left[{ }^{10}\right]$ the following signal model was proposed

$$
v(t)=a_{0}+a_{1} \cos p(t), \quad t \in\left[t_{0}, t_{0}+T\right],
$$

where $T$ is a time interval for which this model is applied. Coefficients $a_{0}$ and $a_{1}$ are constants in this interval and $p(t)$ is generalized phase. Obviously, for a given $v(t)$ and fixed $a_{0}$ and $a_{1}, \cos p(t)$ is defined uniquely. Although these coefficients can be given any value, there is an optimum choice of them. Namely, if $|\cos p(t)| \leq 1$, then $p(t)$ is real and, as shown below, this avoids serious numerical problems. This condition can be met by choosing

$$
\begin{aligned}
& a_{0}=\frac{1}{2}(\max v(t)+\min v(t)), \\
& a_{1}=\frac{1}{2}(\max v(t)-\min v(t)), \quad t \in\left[t_{0}, t_{0}+T\right] .
\end{aligned}
$$

However, we do not assume that this condition is valid; $|\cos p(t)|>1$ will also be allowed. When non-linear transformation $f(x)$ is applied to $v(t)$, we obtain the following result (assuming $f(x)$ to be such that partial sums are certain approximations):

$$
y(t)=f(v(t))=y_{0}+y_{1} \cos p(t)+y_{2} \cos 2 p(t)+\ldots .
$$

Here $y_{k}$ are functions of $a_{0}$ and $a_{1}$, serving as models of the non-linear element. They can be determined once and forever and they do not depend upon the waveform $p(t)$. For many functions, those expansions are well known. As an example, we consider the exponential function, perhaps one of the most important transformations

$$
\exp \left(a_{0}+a_{1} \cos p\right)=e^{a_{0}}\left(I_{0}\left(a_{1}\right)+2 I_{1}\left(a_{1}\right) \cos p+2 I_{2}\left(a_{1}\right) \cos 2 p+\ldots\right),
$$

where $I_{k}(x)$ is modified Bessel function of order $k$. Note that $y_{k}$-s are onedimensional functions.

It is remarkable that calculations related to the waveforms are independent of the non-linear transformation. In consequence, if the same signal is applied to different non-linear elements, there is no need to recalculate the terms $\cos k p$. 


\section{CHEBYSHEV TRANSFORMATION OF WAVEFORMS}

As follows from Eq. (6), the basic calculation step is finding $\cos k p$ from $\cos p$. We do not need to find generalized phase $p(t)$ itself. Moreover, calculation is quite simple because of the well-known relation

$$
\cos k p=T_{k}(\cos p),
$$

where $T_{k}(x)$ is Chebyshev polynomial of the first kind. Therefore, a simple and numerically stable algorithm can be applied for calculating $\cos k p$ :

$$
T_{k+1}(x)=2 x T_{k}(x)-T_{k-1}(x) .
$$

Note that this recursion contains only a single multiplication. We shall call (8) Chebyshev transformation of waveforms and give a short characterization of it.

Chebyshev polynomials are bounded and orthogonal when $|x| \leq 1$ :

$$
\left|T_{k}(x)\right| \leq 1, \quad \int_{-1}^{+1} \frac{1}{\sqrt{1-x^{2}}} T_{i}(x) T_{k}(x) \mathrm{d} x=\delta_{i k} .
$$

It follows that when $|\cos p| \leq 1$ then all $|\cos k p| \leq 1$ with certain benefits for calculations. However, the condition $|\cos p| \leq \eta<1$ does not imply more than $|\cos k p| \leq 1$ and hence there is no need to keep $\cos p$ small - the best is what was declared by (5). Two sets of $\cos k p$ are shown in Figs. 1 and 2 for $\cos p=0.5 \cos t$. Even $\cos p$ is limited by $0.5, \cos 2 p$ reaches -1 and $\cos 3 p$ reaches both -1 and +1 .

Strong bounds on $\cos p$ makes numerical problems simpler but may lead to significantly longer computation time to evaluate min and max of the signals and also due to recalculations caused by changes in $a_{0}$ and $a_{1}$. That is why we consider also the case when $\cos p$ exceeds 1 (since $p$ is a complex function).

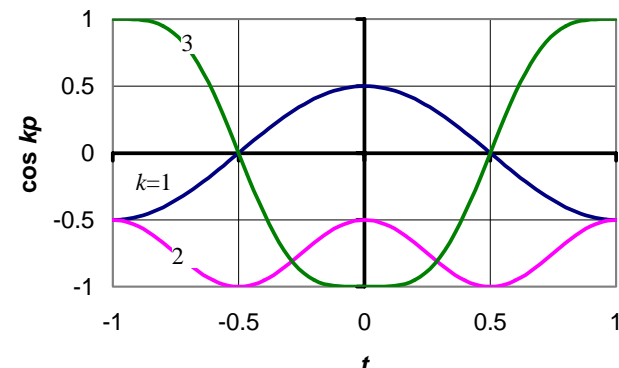

Fig. 1. Waveforms $\cos k p$ when $\cos p=$ $0.5 \cos \pi t$ and $k=1,2,3$.

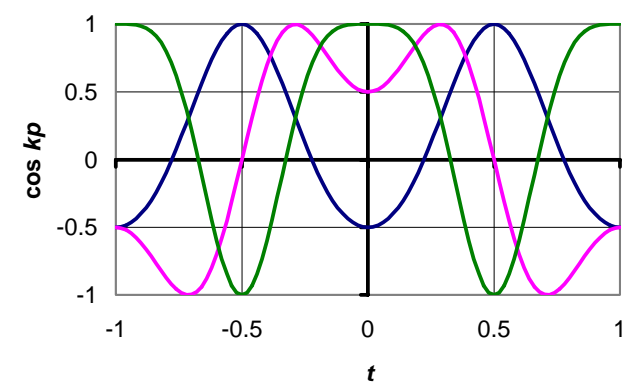

Fig. 2. Waveforms $\cos k p$ when $\cos p=$ $0.5 \cos \pi t$ and $k=4,5,6$. 
Chebyshev polynomials grow monotonically if their argument lays outside the interval $[-1,+1]$. Actually, then we have hyperbolic cosine instead of the trigonometric cosine. Denoting

$$
\cos p=\cosh q
$$

we obtain

$$
\cos k p=\cosh (k \operatorname{Arccosh}(\cosh q))=\cosh k q,
$$

and, consequently, for $\cos p>1$ and $k>>1$ we have

$$
\cosh k q \approx \frac{1}{2} \exp k q
$$

For example, if $\cos p=a \cos t$ and $a \gg>1$, then $\cos k q$ is close to the Gaussian pulse (Fig. 3):

$$
\cosh k q \approx M(a) e^{-\frac{t^{2}}{2 \sigma^{2}}}, \quad M(a) \approx 2^{k-1} a^{k}, \quad \sigma \approx \frac{1}{\sqrt{k}} .
$$

Therefore, if $|\cos p|>1$, then character of the expansion (6) becomes extremely important. If coefficients $y_{k}$ decrease too slowly, then the series may not converge.

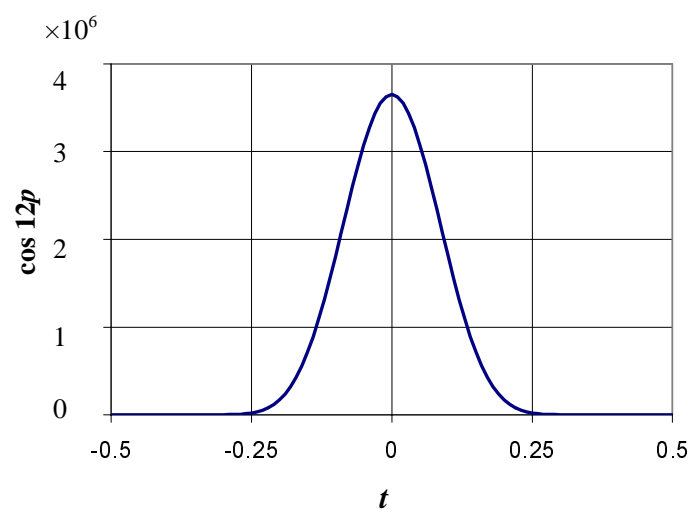

Fig. 3. Waveform $\cos 12 p$; $\cos p=2 \cos \pi t$. 


\section{THE CASE OF THE EXPONENTIAL FUNCTION}

Consider now in greater detail the exponential function $f(v)=\exp (v)$ which has expansion (7). Let us assume that the signal is $v(t)=a \cos t$. Then maximum values of the signal are $\pm a$ and the values of $T_{k}(a)$ have exponential behaviour as in Eq. (13). As these values determine the amplitude of the pulses (Fig. 3), one may expect that spectral components of $T_{k}(v)$ will also show exponential behaviour. This is confirmed by calculations as shown in Fig. 4. Higher harmonics exhibit similar behaviour and the number of efficient harmonics (with significant contribution to the sum) is about $k$ (since $T_{k}$ is a polynomial).

Asymptotic behaviour of Bessel functions for a fixed argument and varying index is known $\left[{ }^{14}\right]$. A simple but sufficient description is

$$
\ln I_{v}(x) \sim B-C v-D v^{2},
$$

where $B, C$, and $D$ are constants.

As $T_{v}$ is exponential in $v$, then $\ln T_{v}$ is linear in $H+E v$ and for multiplicative terms in Eq. (7) we have

$$
\ln \left[I_{v}(\cdot) T_{v}(\cdot)\right]=B+H+(E-C) v-D v^{2} .
$$

It follows that the last expression has a maximum and its form as a function of $v$ is close to the Gaussian curve. An example is given in Fig. 5.

Obviously, after the properties of the curve have been identified, it is sufficient to calculate only significant values of $I_{v} T_{v}$ around the maximum.

In fact, we do not calculate values of the signal, but components of its spectrum. However, as mentioned above, the components of the spectrum have the same behaviour as maximum values. Therefore the above conclusions are valid also for the spectra.

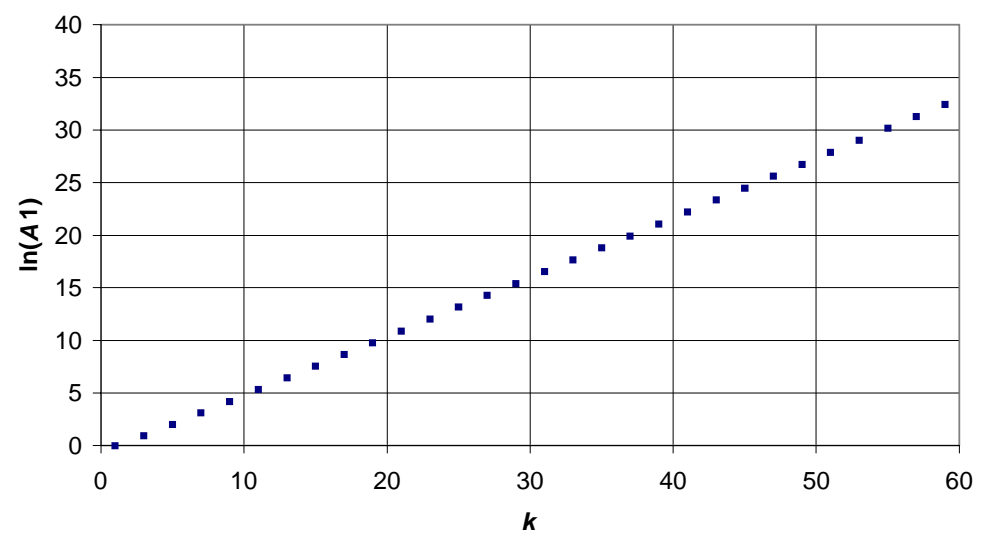

Fig. 4. Logarithm of the first harmonic $A_{1}$ of $T_{k}(2 \cos t)$ as a function of $k$. 


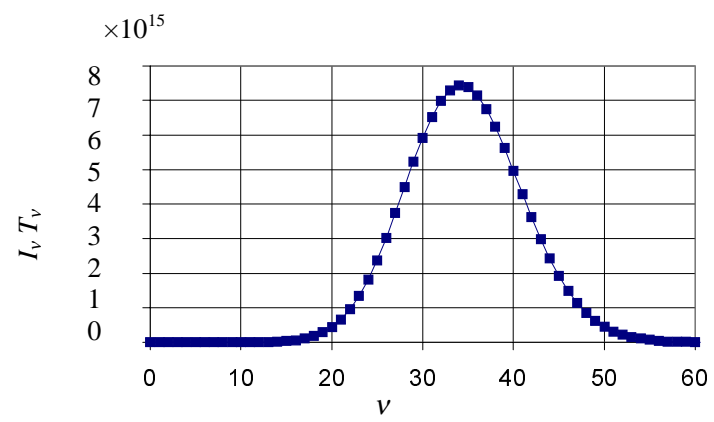

Fig. 5. Behaviour of $I_{v} T_{v}$ as a function of $v$.

Expansion (7) is based on the representation of the exponent as $\left[{ }^{15}\right]$

$$
e^{a x}=I_{0}(a)+2 \sum_{k=1}^{\infty} I_{k}(a) T_{k}(x),
$$

which converges for $|x| \leq 1$, since Chebyshev polynomials are orthogonal on $[-1,+1]$. As shown earlier, it actually converges also outside this interval. However, because of alternating signs, it is practically impossible to use it for negative values of $x$. When computing spectra, we do not encounter this problem.

Equation (16) shows that our model is based on the Chebyshev expansion of non-linear functions. This is the result of our cosine-based signal model. Consequently, the magnitude is strictly related to the interval $[-1,+1]$. Exceeding these limits may cause problems; for example, expansion of non-smooth functions does not converge outside this interval. One could use another approach like choosing another fundamental set of functions and applying it to $\cos p$. For example, assume that weighing function $w(x)>0$ determines a set of orthogonal polynomials $P_{k}(x)$. Then we have the expansion

$$
f(\cos p)=f_{0}+\sum_{k=1}^{\infty} f_{k} P_{k}(\cos p),
$$

and if truncated, it is a polynomial in $\cos p$. For a fixed $k$, one can rearrange the terms and obtain again the expansion in the form

$$
f(\cos p)=\bar{f}_{0}+\sum_{k=1}^{\infty} \bar{f}_{k} \cos k p
$$

For example, weighing function $\exp \left(-x^{2}\right)$ determines Hermite polynomials $H_{k}(x)$ on $[-\infty,+\infty]\left[{ }^{14}\right]$. They are used in the Wiener model of non-linear systems. Hermite polynomials can also be calculated by a simple recursion and expressed as

$$
\begin{gathered}
H_{k+1}(x)=2 x H_{k}(x)-2 k H_{k-1}(x), \\
H_{0}=T_{0}(x), H_{1}(x)=2 T_{1}(x), \quad H_{2}(x)=2 T_{2}(x), H_{3}(x)=2 T_{3}(x)-6 T_{1}(x), \ldots
\end{gathered}
$$


It may look as if using Hermite polynomials will solve the problems related to the boundaries. However, the weighing function decreases rapidly when $|x|>3$. For example, $\operatorname{sign}(x)$ has an extremely slowly converging Chebyshev expansion for $-1<x<+1$ and does not converge for $|x|>1$. Hermite expansion is converging everywhere, but very slowly, and error may be very large when the truncated expansion is used. We do not consider other representations in this paper.

\section{EXTENDING FOURIER SERIES}

Return now to the signal form (4), assuming for simplicity that $t_{0}=0$

$$
v(t)=a_{0}+a_{1} \cos p(t), \quad t \in[0, T] .
$$

To consider linear dynamical aspects, we concentrate on $\cos p(t)$ as $a_{0}$ and $a_{1}$ can be taken equal to 0 and 1 , respectively.

As our interval of interest is $[0, T]$, we can extend $\cos p(t)$ outside this interval to meet any of our goals. Since the object is an oscillating system, it should be acceptable to assume similarity of waveforms in neighbouring intervals, for example in $[0, T]$ and $[T, 2 T]$, or $[T, 2 T+\varepsilon]$. That would mean almost periodic signals. On the other hand, harmonic waveforms are extremely simply processed by linear systems and therefore choosing sinusoids as building blocks, we may significantly reduce complexity of the calculations.

Obviously, we could also use Fourier series

$$
\cos p(t)=\sum_{k=0}^{\infty}\left(b_{k}^{\mathrm{R}} \cos k \omega t-b_{k}^{\mathrm{I}} \sin k \omega t\right), \quad \omega=\frac{2 \pi}{T} .
$$

However, using truncated series will result in equal values of the function at both ends of the period, which is acceptable only in steady state solutions, but not for transients. To build a waveform that uses finite number of harmonics and allows different values at the ends of the period, we replace (21) by

$$
\cos p(t)=b_{0}+b_{1 / 2}^{\mathrm{R}} \cos \frac{\omega}{2} t-b_{1 / 2}^{\mathrm{I}} \sin \frac{\omega}{2} t+\sum_{k=1}^{n}\left(b_{k}^{\mathrm{R}} \cos k \omega t-b_{k}^{\mathrm{I}} \sin k \omega t\right),
$$

that includes half-frequency components. These make it possible that the signal and its first derivative have different values at the ends of the period:

$$
\begin{array}{ll}
\cos p(0)=+b_{1 / 2}^{\mathrm{R}}+b_{0}+\sum_{k=1}^{n} b_{k}^{\mathrm{R}}, & \cos p(T)=-b_{1 / 2}^{\mathrm{R}}+b_{0}+\sum_{k=1}^{n} b_{k}^{\mathrm{R}}, \\
\cos ^{\prime} p(0)=-b_{1 / 2}^{\mathrm{I}} \frac{\omega}{2}-\sum_{k=1}^{n}\left(b_{k}^{\mathrm{I}} k \omega t\right), & \cos ^{\prime} p(T)=+b_{1 / 2}^{\mathrm{I}} \frac{\omega}{2}-\sum_{k=1}^{n}\left(b_{k}^{\mathrm{I}} k \omega t\right) .
\end{array}
$$




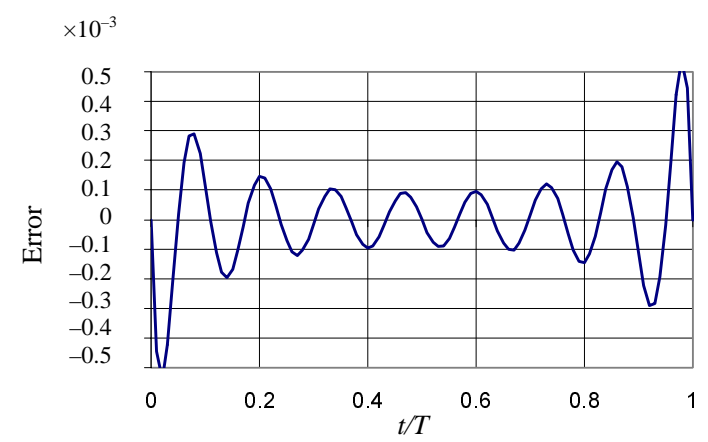

Fig. 6. Approximation error of Eq. (24), $n=7$.

Including half-frequency components solves also another problem. When integrating the constant term in Eq. (22), we obtain a linear term that is not a part of the general form of Eq. (22). However, linear term can be approximated with very high accuracy by the half-frequency component:

$$
t \approx \frac{T}{2}\left(1-\cos \frac{\omega}{2} t\right)+\frac{T}{\pi} \sum_{k=1}^{n}\left(\frac{1}{k\left(4 k^{2}-1\right)}\right) \sin k \omega t .
$$

Figure 6 shows approximation error of Eq. (24) when $n=7$.

\section{INTEGRATION FORMULA}

Integrating (22) starting from $t=0$ and denoting $\cos p(t)=u(t)$, we obtain

$$
\begin{aligned}
\int_{0}^{t} u(\tau) \mathrm{d} \tau=u(0) & +b_{0} t+\frac{T}{\pi} b_{1 / 2}^{\mathrm{R}} \sin \frac{\omega}{2} t+\frac{T}{\pi} b_{1 / 2}^{\mathrm{I}}\left(\cos \frac{\omega}{2} t-1\right) \\
+ & \sum_{k=1}^{n} \frac{1}{k \omega}\left(b_{k}^{\mathrm{R}} \sin k \omega t+b_{k}^{\mathrm{I}}(\cos k \omega t-1)\right) .
\end{aligned}
$$

Let the integrator be a capacitor with capacitance $C$, integrating current $i(t)$, and resulting voltage $v(t)$. Applying Eq. (24), substituting $i(0)=u(0)=\cos p(0)$ from Eq. (23) and reordering terms, we obtain

$$
\begin{aligned}
v(t)=v(0) & +\frac{T}{2 C} i_{0}+\sum_{k=1}^{n} \frac{T}{2 \pi C k} i_{k}^{\mathrm{I}}+\left(\frac{T}{\pi C} i_{1 / 2}^{\mathrm{I}}-\frac{T}{2 C} i_{0}\right) \cos \frac{\omega}{2} t-\left(-\frac{T}{\pi C} i_{1 / 2}^{\mathrm{R}} \sin \frac{\omega}{2} t\right) \\
& +\sum_{k=1}^{n}\left[\frac{T}{2 \pi C k} i_{k}^{\mathrm{I}} \cos k \omega t-\left(-\frac{T}{2 \pi C k} i_{k}^{\mathrm{R}}-\frac{T}{\pi k C\left(4 k^{2}-1\right)} i_{0}\right) \sin k \omega t\right] .
\end{aligned}
$$


Comparing Eq. (26) with

$$
v(t)=v_{0}+v_{1 / 2}^{\mathrm{R}} \cos \frac{\omega}{2} t-v_{1 / 2}^{\mathrm{I}} \sin \frac{\omega}{2} t+\sum_{k=1}^{n}\left(v_{k}^{\mathrm{R}} \cos k \omega t-v_{k}^{\mathrm{I}} \sin k \omega t\right),
$$

which is obtained from Eq. (22), we get the following set of equations where the second index denotes the step number:

$$
\begin{aligned}
& v(0)=v_{0, n}+v_{1 / 2, n}^{\mathrm{R}}+\sum_{k=1}^{\infty} v_{k, n}^{\mathrm{R}}=v_{0, n-1}-v_{1 / 2, n-1}^{\mathrm{R}}+\sum_{k=1}^{\infty} v_{k, n-1}^{\mathrm{R}}, \\
& i_{1 / 2, n}^{\mathrm{R}}=-\frac{\pi C}{T} v_{1 / 2, n}^{\mathrm{I}}, \quad i_{1 / 2, n}^{\mathrm{I}}=\frac{\pi C}{T} v_{1 / 2, n}^{\mathrm{R}}+\frac{\pi}{2} i_{0, n}, \\
& i_{k, n}^{\mathrm{R}}=-\frac{2 \pi k C}{T} v_{k, n}^{\mathrm{I}}-\frac{2}{4 k^{2}-1} i_{0, n}, \quad i_{k, n}^{\mathrm{I}}=\frac{2 \pi k C}{T} v_{k, n}^{\mathrm{R}} .
\end{aligned}
$$

The first equation expresses the continuity of voltage, the others are related only to the current integration step. The set of expressions (28) provides the algorithm of the EFS formula.

Upper indexes R (real) and I (imaginary) are used to emphasize that complex numbers can be applied for the implementation of the algorithm:

$$
\begin{aligned}
& I_{k}=i_{k, n}^{\mathrm{R}}+j i_{k, n}^{\mathrm{I}}, \quad V_{k}=v_{k, n}^{\mathrm{R}}+j v_{k, n}^{\mathrm{I}}, \\
& I_{k}=j k \omega C V_{k}+I_{0}, \\
& I_{0}=-\frac{2}{4 k^{2}-1} i_{0}, \quad k \geq 1, \\
& I_{0}=j \frac{\pi}{2} i_{0}, \quad k=\frac{1}{2} .
\end{aligned}
$$

Equations (29) make it possible to use the classical ac analysis sequentially at all frequencies.

\section{ANALYSIS OF THE INTEGRATION FORMULA}

We evaluate the integration formula as usually, by applying it to the linear problem

$$
\frac{\mathrm{d} v(t)}{\mathrm{d} t}=\lambda v(t)
$$

with exact solution

$$
v(t)=v(0) e^{\lambda t}
$$


As our integration step is $T$, we evaluate the value of $\rho=v(T) / v(0)$ and conclude that the correct value of $\rho$ is $\exp (\mu)$ with $\mu=\lambda T$.

From (28) we obtain

$$
\begin{aligned}
& v(0)=v_{0, n}+v_{1 / 2, n}^{\mathrm{R}}+\sum_{k=1}^{\infty} v_{k, n}^{\mathrm{R}}, \\
& v(T)=v_{0, n}-v_{1 / 2, n}^{\mathrm{R}}+\sum_{k=1}^{\infty} v_{k, n}^{\mathrm{R}} .
\end{aligned}
$$

Thus

and, finally

$$
\begin{aligned}
& v_{1 / 2}^{\mathrm{R}}=-\frac{\pi}{2} \frac{\mu}{\pi^{2}+\mu^{2}} v_{0}, \\
& v_{k}^{\mathrm{R}}=-\frac{2 \mu^{2}}{\left(4 k^{2}-1\right)\left[(2 k \pi)^{2}+\mu^{2}\right]} v_{0},
\end{aligned}
$$

$$
c_{1 / 2}=-\frac{\pi}{2} \frac{\mu}{\pi^{2}+\mu^{2}}, \quad c_{k}=-\frac{2 \mu^{2}}{\left(4 k^{2}-1\right)\left[(2 k \pi)^{2}+\mu^{2}\right]} .
$$

A remarkable property of this formula is that it maps the imaginary axis from the $\mu$-plane onto a unit circle in the $\rho$-plane. This means that the formula exactly preserves the stability.

On the other hand, for a fixed number of harmonics $n, \rho \rightarrow 1$ when $|\mu| \rightarrow \infty$. This means that fast components may not decrease when large integration step is applied. However, the most popular trapezoidal method has similar property - fast components may create saw-tooth oscillations, well known to designers. To characterize the EFS formula, we first consider some special cases.

Case 1. Real poles. Behaviour of a real negative pole is shown in Fig. 7. First, $\rho$ has been calculated from the EFS formula using $n=12$ and the result is mapped back to the $\mu$-plane. It is clearly visible that on the left of $\mu \approx-7$, the mapped values differ from the correct ones and saturate at about $\mu=-8$. In fact, there exists a maximum value of the pole that cannot be exceeded (the effect described above). It follows that the step size is limited and further we show that the value close to -8 is critical even, for a much larger number of harmonics. 


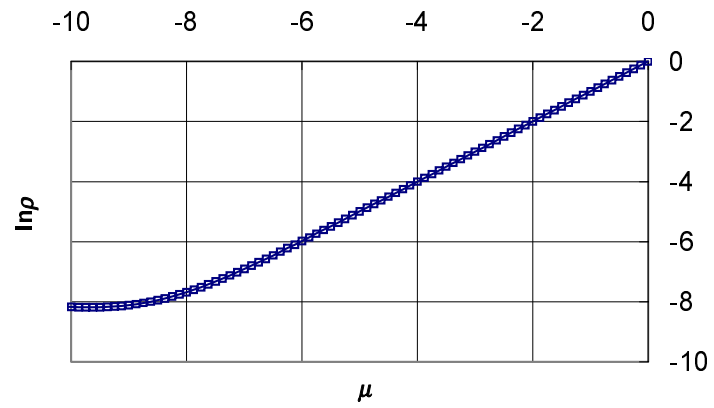

Fig. 7. Mapping real poles, $n=12$.

Case 2. Imaginary poles. These are mapped correctly back to the imaginary axis and according to our construction, the EFS formula is correct for a certain number of harmonics. In Fig. 8, the relative mapping error is shown. One can see that for intermediate frequencies the error may be remarkable, besides, increasing $n$ reduces the error.

Case 3. Complex poles. Consider poles located on a circle (Fig. 9). When radius of the circle is small, the mapping is correct. To make the errors observable, we use a large radius.

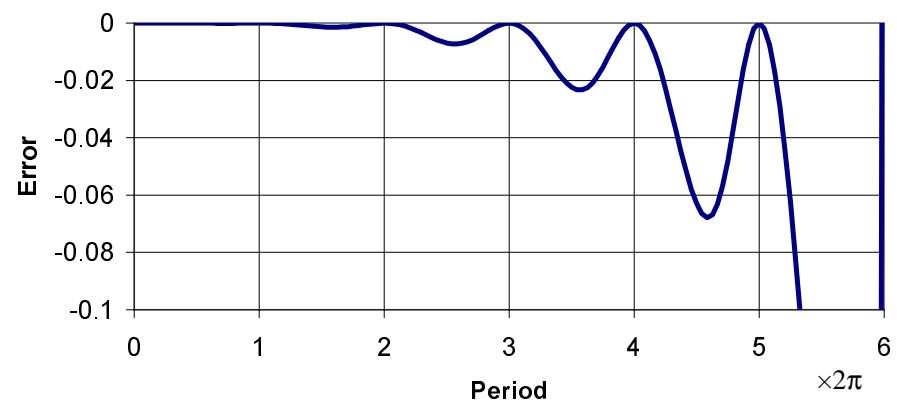

Fig. 8. Mapping imaginary poles.

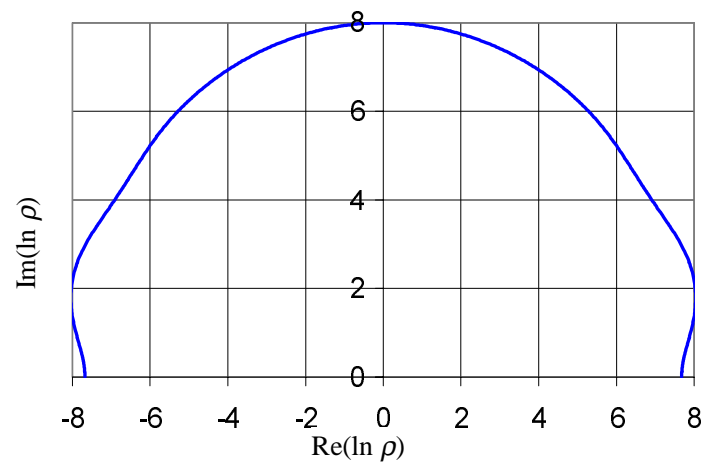

Fig. 9. Mapping a circle, $r=8.0$. 
Mapping of a circle with $r=8$ in Fig. 9 shows again that if the real part of $\ln \rho$ is more than 6-7, remarkable distortions appear. We shall explain the reasons of such behaviour in the next section.

\section{ZEROES AND PADÉ EXPANSIONS}

EFS formula is very close to the Padé fractions of the exponent $\left[{ }^{16}\right]$. We are going to show what is common to them and what are the differences. First, note that the EFS formula has the same structure as the diagonal Padé fraction:

$$
\rho=\frac{P\left(\mu^{2}\right)-\mu Q\left(\mu^{2}\right)}{P\left(\mu^{2}\right)+\mu Q\left(\mu^{2}\right)},
$$

where $P\left(\mu^{2}\right)$ and $Q\left(\mu^{2}\right)$ are polynomials.

Such a structure maps the imaginary axis always back to itself, ensuring good stability. The Padé expansions of the exponent have been extensively studied, including distribution of the zeroes. This is not a traditional way to characterize integration formulas, but in our case it appears useful by explaining the effects described above.

Note that trapezoidal formula (that is not the case of EFS) is a diagonal Padé fraction:

$$
\rho=\frac{1+\frac{1}{2} \mu}{1-\frac{1}{2} \mu}
$$

Compare now the second order fractions (EFS uses only the half-frequency component):

$$
\rho=\frac{1+\frac{1}{2} \mu+\frac{1}{12} \mu^{2}}{1-\frac{1}{2} \mu+\frac{1}{12} \mu^{2}}, \quad \rho=\frac{1+\frac{1}{2} \mu+\frac{1}{\pi^{2}} \mu^{2}}{1-\frac{1}{2} \mu+\frac{1}{\pi^{2}} \mu^{2}} .
$$

Difference in the coefficients is about $10 \%$. This is explained by the fact that while the Padé expansion has 5 first terms in the Taylor series equal to those of the exponent, EFS has only 2. When order of the function increases, first coefficients of the EFS formula become closer to those of the Padé expansion, but they are always different because of different approximation. Let us compare also zeroes of the functions (37) (poles are located symmetrically in the right half plane): 


$$
\begin{aligned}
& z_{1,2}=-3 \pm j \sqrt{3}=-3.00000 \pm j 1.73205, \quad \text { Padé } \\
& z_{1,2}=-\frac{\pi^{2}}{4} \pm j \sqrt{\frac{16 \pi^{2}-\pi^{4}}{16}}=-2.46740 \pm j 1.94462 . \quad \text { EFS }
\end{aligned}
$$

Second order is very low, EFS uses only the half-frequency component. Therefore this formula has no unit value on the imaginary axis, i.e., it is not able to carry out integration with a step equal to a period. However, location of zeroes shows a general property - EFS has zeroes closer to the imaginary axis. This has important consequences as will be shown below.

Let us consider the case $n=11$ that produces 24 th order fractions. For comparison, Fig. 10 shows location of poles for both EFS and Padé expansions (zeroes are located symmetrically in the left half-plane). One can again see that poles of EFS are located closer to the imaginary axis but have a wider distribution along that axis. Maximum real parts are located near $\operatorname{Re}(p)=9$ and that is just the reason why EFS fails when $\mu$ approaches this region. This limit increases together with the order, but extremely slowly: doubling the number of harmonics increases the limit only by about 1.0.

Padé approximation processes real poles much better, but its behaviour on the imaginary axis is less accurate as will be shown below.

For the same case the location of points where $\rho=1$ (step equals to the full period, $2 k \pi$ ) and $\rho=-1$ (odd multiple of the half-period, $(2 k+1) \pi$ ) is shown in Table 1 where the values are multiples of $\pi$. One can see exact location of the

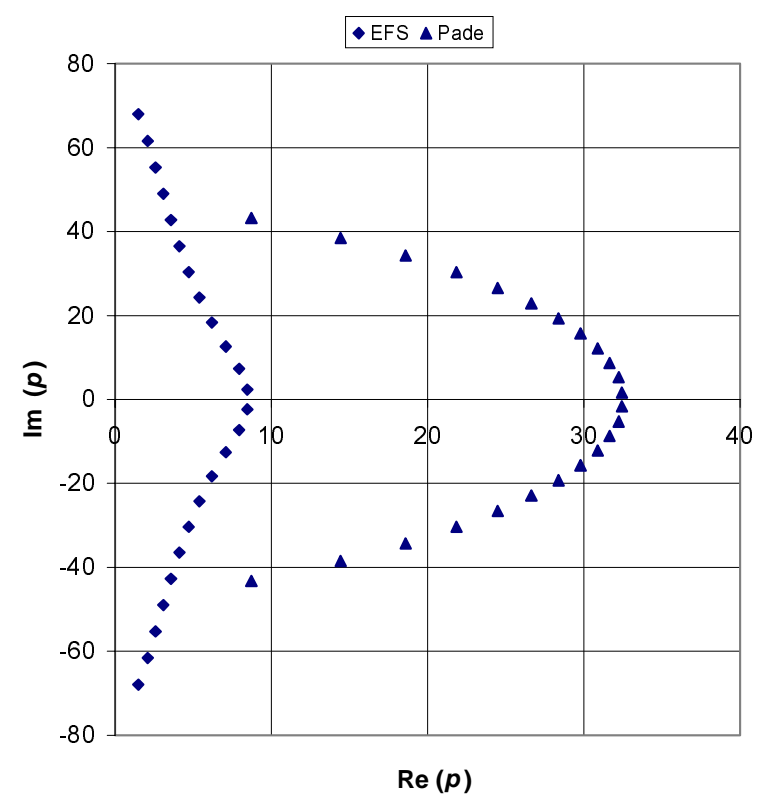

Fig. 10. Zeroes of EFS and Padé fractions, order is 24. 
Table 1. Location of the points $\rho= \pm 1$ on the imaginary axis

\begin{tabular}{c|c|r|r}
\hline \multicolumn{2}{c|}{$\rho=1$} & \multicolumn{2}{c}{$\rho=-1$} \\
\hline EFS & \multicolumn{1}{c|}{ Padé } & \multicolumn{1}{c}{ EFS } & \multicolumn{1}{c}{ Padé } \\
\hline 2.00000000 & 2.00000000 & 1.00000000 & 1.00000000 \\
4.00000000 & 4.00000000 & 3.00026859 & 3.00000000 \\
6.00000000 & 6.00000000 & 5.00136886 & 5.00000000 \\
8.00000000 & 8.00000000 & 7.00394813 & 7.00000000 \\
10.00000000 & 10.00000175 & 9.00881901 & 9.00000003 \\
12.00000000 & 12.00096293 & 11.01709133 & 11.00005495 \\
14.00000000 & 14.06067256 & 13.03041533 & 13.00981969 \\
16.00000000 & 16.71206092 & 15.05148743 & 15.24456543 \\
18.00000000 & 21.33488815 & 17.08526838 & 18.65534969 \\
20.00000000 & 31.07896214 & 19.14262777 & 25.18742511 \\
22.00000000 & 61.12543443 & 21.25655039 & 41.03280973 \\
& & 27.56569900 & 121.75075779
\end{tabular}

EFS points for $\rho=1$; less correct are the EFS points for $\rho=-1$ while the Padé fraction has larger errors at higher values.

Figure 11 shows the function $\rho(w)$, which in this case is equal to $\cos (w)$. One can see that up to the 11th period, EFS exactly estimates the location of the value 1.0. However, values -1.0 are located with remarkable error starting with 8.5. The Padé approximation deviates from the cosine function after the 7 th period.

Qualitative behaviour of EFS can be explained as follows. The formula is designed to describe oscillating systems and hence it has high precision when integration step is a multiple of the period corresponding to the near-imaginary (dominant) pole. Its behaviour in case of real poles is poor, because non-periodic components are represented only by half-frequency components. However, this is of importance only by using very large integration steps that is usually not applicable, for example, in case of the Gear formulas.

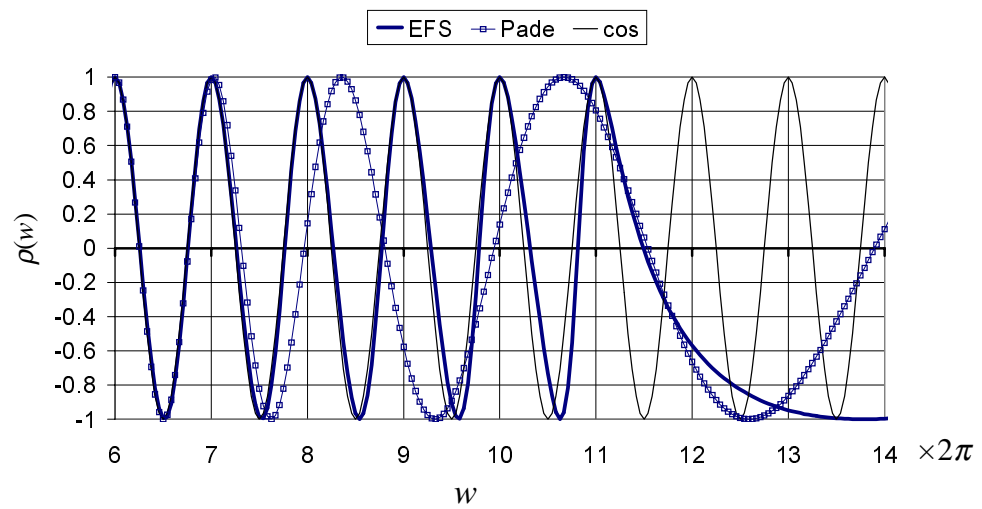

Fig. 11. EFS, Padé fraction, and cosine for $n=11$. 


\section{EXAMPLE}

In this paper, we shall not discuss implementation of the above method. However, we shall describe a simple benchmark problem $\left[{ }^{10}\right]$. Consider a simple circuit shown in Fig. 12. Non-linear two-pole is described by the following equation:

$$
i=0.1\left(e^{\frac{v}{0.27}}-1\right)
$$

This is a simplified case as components required for implementation of the oscillator are replaced by a negative resistance. Consequently, no chaos is possible in that circuit. If one applies harmonic linearization to that circuit, the following result is obtained: voltage is $0.8214 \mathrm{~V}$ and the period of oscillation $T=2 \pi$. Both are close to real values with an error of about $0.5 \%$.

Applying EFS, we can set $v_{0}=0, v_{1}=1.0$ and keep them constant because the dc component should be zero and the ac component will not exceed 1.0, as seen from the rough solution.

The number of harmonics was varied from 7 to 16 , the number of terms in the expansion of the exponential function - from 7 to 11 without remarkable changes in results. As the circuit is autonomous, the period control (detection) was implemented as follows. Period can be determined as the distance between equal values of the signal. This condition can be used when half-frequency cosine is zero. This condition was inserted into equations which solve the problem. Modelling included the start-up of the oscillator and, after reaching the steady state, instantaneous changing of the capacitor value by $1 \%$. The results are shown in Figs. 13-15.

As the method is applied to every integration step, it is possible to follow the period values in time. Figure 14 shows a rapid change of the oscillation period when the oscillation amplitude becomes stabilized. Such effect is typical for start-up processes, demonstrating that the frequency reaches final value before its amplitude reaches a steady state. Instantaneous change of the period value as a reaction to the change of $C$ is correctly modelled as seen in Fig. 15.

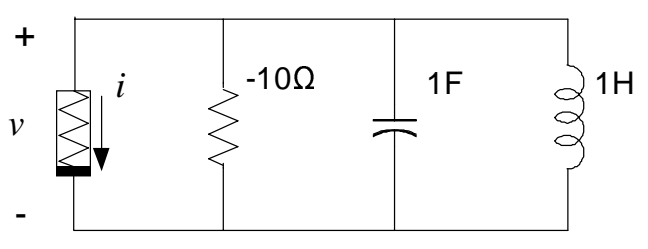

Fig. 12. Test circuit. 


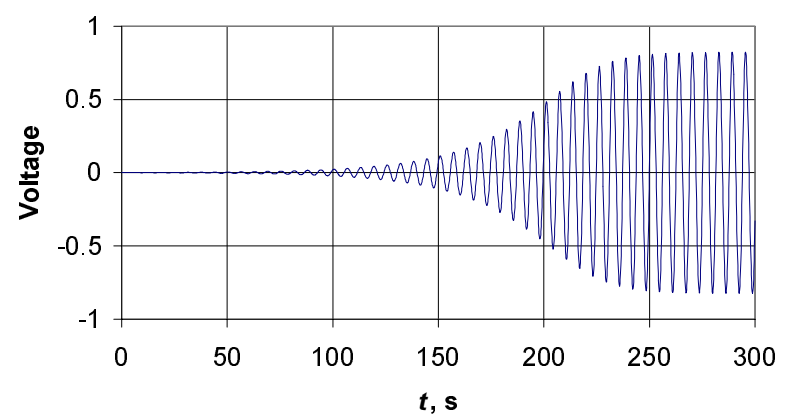

Fig. 13. The start-up process; voltage is shown in relative units.

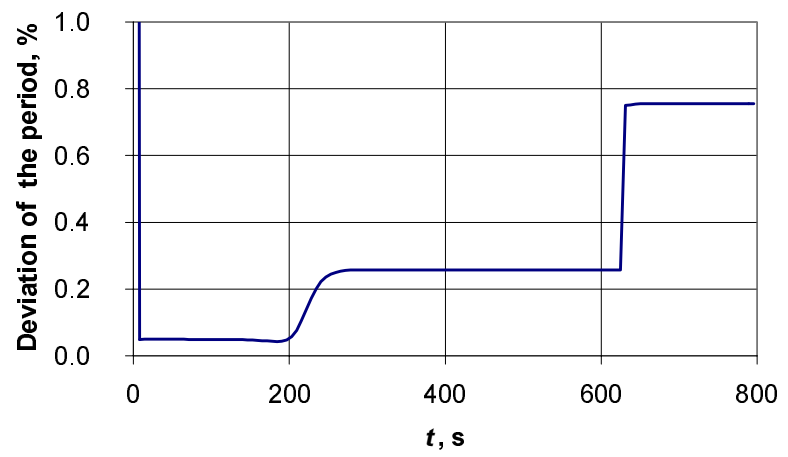

Fig. 14. Deviation of the period from $2 \pi$; change of $C$ at $t=628 \mathrm{~s}$.

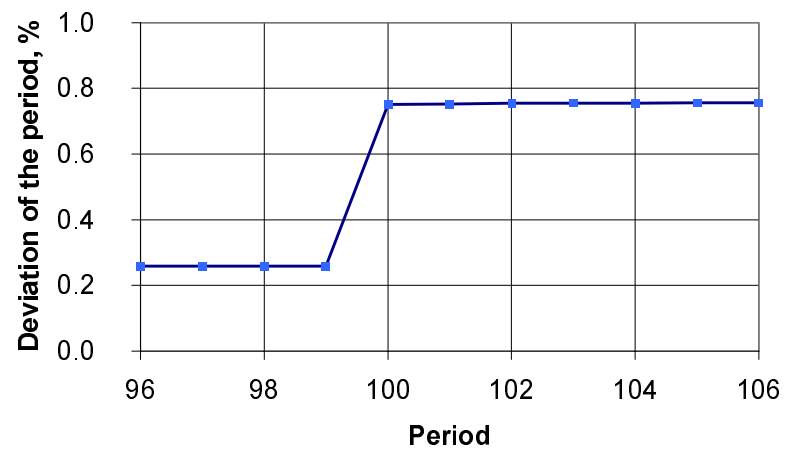

Fig. 15. Change of the period after the change of $C$.

\section{CONCLUSIONS}

Properties of the new modelling algorithm EFS based on the extended Fourier series have been studied. It is shown that the integration formula is well suited to modelling narrow-band systems. It is correct even when a very large integration 
step is used, if it is synchronized with the oscillation. However, EFS formula does not allow very large steps along the real axis. It is shown that the EFS formula is close to the Padé fraction.

Signals are combined from harmonics and new components can be added without loss of stability if the accuracy is not sufficient. The integration method allows to model autonomous systems since the period can be detected during solution.

For non-linear components a representation that is based on the Chebyshev expansion of non-linear functions is proposed. This approach allows to divide the non-linear model into a function of two variables and an independent waveform transformation. It is shown that the Chebyshev expansion can be used also outside its standard convergence region.

Implementation problems are not considered in this paper. There is a lot of work to be done to optimize the iteration process, Chebyshev transformation and overall organization of the calculations as the method uses many variables.

\section{REFERENCES}

1. Kennedy, M. P. Chaos in the Colpitts oscillator. IEEE Trans. CAS, 1994, 41, 771-774.

2. Kukk, V. Harmonic linearisation for oscillator design. In Proc. 5th International Conference on VLSI CAD (ICVC'97). Seoul, 1997, 472-474.

3. Irscheid, O. and Blum, A. Phase noise in oscillators - numerical calculations using SPICE. In Proc. European Conference on Circuit Theory and Design (ECCTD'97). Budapest, 1997, 462-467.

4. Brambilla, A. and Maffezzoni, P. Envelope following method for the transient analysis of electrical circuits. IEEE Trans. CAS, 2000, 47, 999-1008.

5. Kukk, V. Modelling transients in oscillator circuits. In Proc. 6th International Workshop on Nonlinear Dynamics of Electronic Systems (NDES'97). Moscow, 1997, 520-525.

6. Kukk, V. Fast modelling of oscillator transients. In Proc. Electronic Circuits and Systems Conference (ECS'97). Bratislava, 1997, 27-30.

7. Kukk, V. Large signal models for oscillator design. In Proc. International Symposium on Circuits and Systems (ISCAS'98). Monterey, 1998. CD-ROM.

8. Brachtendorf, H. G., Welsch, G., and Laur, R. A time-frequency algorithm for the simulation of the initial transient response of oscillators. In Proc. International Symposium on Circuits and Systems (ISCAS'98). Monterey, 1998. CD-ROM.

9. Heikkilä, P., Valtonen, M., and Veijola, T. Harmonic balance of nonlinear circuits with multitone excitation. In Proc. European Conference on Circuit Theory and Design (ECCTD'91). Copenhagen, 1991, 802-811.

10. Kukk, V. Large signal models and monotonic phase signals for modeling of oscillators. In Proc. 7th International Workshop on Nonlinear Dynamics of Electronic Systems (NDES'99). Rønne, Denmark, 1999, 103-106.

11. Kukk, V. Large time-step integration method for modeling of oscillating circuits. In Proc. IEEE International Symposium on Circuits and Systems (ISCAS'2000). Geneva, 2000. CD-ROM.

12. Kukk, V. A large signal integration formula. In Proc. First IEEE Balkan Conference on Signal Processing, Communications, Circuits, and Systems. Istanbul, 2000. CD-ROM.

13. Kukk, V. Large signal integration formula based on truncated Fourier series. In Proc. 43rd IEEE Midwest Symposium on Circuits and Systems. Lansing, MI, 2000. CD-ROM.

14. Bateman, H. and Erdélyi, A. Higher Transcendental Functions. McGraw-Hill, New York, 1953. 
15. Luke, Y. L. Mathematical Functions and Their Approximations. Academic Press, New York, 1975.

16. Driver, K. A. and Temme, N. M. On Polynomials Related with Hermite-Pade Approximations to the Exponential Function. Report MAS-R9716, CWI, Amsterdam, 1997.

\title{
MITTELINEAARSETE SÜSTEEMIDE MODELLEERIMINE LAIENDATUD FOURIER' REA ABIL
}

\begin{abstract}
Vello KUKK
Tugevasti ostsilleeruvaid alamsüsteeme omavate mittelineaarsete süsteemide modelleerimiseks on esitatud meetod, mis põhineb signaalide kirjeldamisel poolsagedusega komponenti sisaldava laiendatud Fourier' reana. Seetõttu osutub võimalikuks modelleerida siirdeprotsesse ning kasutada harmoonilist lineariseerimist, mis realiseeritakse Tšebõševi signaaliteisenduse abil. Nende võtetega saab mittelineaarse elemendi kirjelduse lahutada kaheks sõltumatuks komponendiks. Saadud integreerimismeetodil on väga head stabiilsusomadused ning selle täpsus on lihtsalt reguleeritav kasutatavate harmooniliste arvuga.
\end{abstract}

